\title{
Evaluation of Few-Layer Graphene Grown by Gas-Source Molecular Beam Epitaxy Using Cracked Ethanol*
}

\author{
Fumihiko Maeda ${ }^{\dagger}$ and Hiroki Hibino \\ NTT Basic Research Laboratories, Nippon Telegraph and Telephone co., \\ 3-1 Morinosato-Wakamiya, Atsugi-shi, Kanagawa 243-0198, Japan \\ Ichiro Hirosawa and Yoshio Watanabe \\ The Japan Synchrotron Radiation Research Institute, \\ 1-1-1 Kouto, Sayo-cho, Sayo-gun, Hyogo 679-5198, Japan \\ (Received 22 October 2010; Accepted 14 January 2011; Published 26 February 2011)
}

\begin{abstract}
To evaluate graphene grown by a new method based on gas-source molecular beam epitaxy (MBE), in which a cracked-ethanol source is employed, from the macroscopic viewpoint, we investigated crystal truncation rod scattering by x-ray reflectivity measurement. From the analysis of the x-ray reflectivity data, we found that the MBE-grown graphene forms a layered atomic structure from the macroscopic view, too. The average spacing of the MBE-grown graphene was $3.39 \AA$, which is larger than the interlayer spacing values for bulk crystalline graphite, and the height distributions of the MBE-grown graphene were relatively large. The occupancies of the graphene are smaller than unity and are smaller than that of its underlying graphene, suggesting that graphene did not grow in a layer-by-layer manner but in a three dimensional one, while each layer grew laterally. These results indicate that our new approach is feasible for the formation of wafer-scale graphene, although further improvement of the quality of the graphene by optimizing the growth condition is needed. [DOI: 10.1380/ejssnt.2011.58]
\end{abstract}

Keywords: X-ray scattering, diffraction, and reflection; Growth; Nano-films, stacks, and other nano materials; Graphene; $\mathrm{SiC}(0001) ; \mathrm{MBE}$

\section{INTRODUCTION}

Because of the excellent electrical performance of fewlayer graphene (FLG), it has attracted much attention as an electronics material for the next generation of devices. However, the performance of FLG was demonstrated using small micrometer-size pieces of FLG exfoliated from bulk graphite [1] and they are not suitable for large-scale manufacturing. Hence, establishing alternative ways of forming wafer-scale FLG is of great interest from the viewpoint of large-scale integration.

Many researchers have been looking at graphene formation by means of epitaxy on $\mathrm{SiC}[2]$ or on transition metals $[3,4]$ as alternative processes, which are based on familiar phenomena in the surface science field. However, in these methods, the significant interaction between the FLG and substrates restricts the application of the FLG in the as-grown condition. Hence, the research target for this graphene is shifting to finding ways to reduce the interaction by the intercalation of atoms, such as hydrogen [5] and gold [6], in the case of the $\mathrm{SiC}$, and to transfer it onto another substrate [7] in the case of transition metals. Thus, those methods are closest to practical use at present but still under investigation.

Recently, some new alternative approaches to obtaining graphene have been proposed. We have proposed a new method based on gas-source molecular beam epitaxy (MBE), in which a cracked-ethanol source is employed [8]. This growth method allows us to independently con-

\footnotetext{
*This paper was presented at the 6th International Workshop on Nano-scale Spectroscopy and Nanotechnology (NSS-6), Kobe University Centennial Hall, Kobe, Japan, 25-29 October, 2010.

${ }^{\dagger}$ Corresponding author: maeda.f@lab.ntt.co.jp; Present address: JST-ERATO, 3-2-1 Sakado, Takatsu-ku, Kawasaki-shi, Kanagawa 213-0012, Japan
}

trol the substrate temperature and the supply of growth material. This could lead to growth mode control in a layer-by-layer manner at high temperature, which would result in high-quality and large-domain growth. In our previous study, we showed the feasibility of this growth method through the atomic layer structure of the grown graphene observed by transmission electron microscopy (TEM). However, since the idea of using TEM to macroscopically evaluate the grown thin layer is practically unimaginable. In this study, we evaluated the graphene grown by our new method using crystal truncation rod (CTR) scattering from the macroscopic viewpoint.

\section{EXPERIMENTAL}

For the growth, an ultrahigh-vacuum growth system was equipped with an ethanol-gas supply system with a cracking unit consisting of a $\mathrm{W}$ filament. Cracked ethanol was supplied to the substrates, with its flow rate controlled with a mass flow controller. Effective growth species derived from the cracked ethanol was extrapolated to be ethylene from the activation energy estimated by the dependence of $\mathrm{W}$-filament temperature on the graphiticmaterial deposition rate [9]. Because we expected homoepitaxial growth, we used graphene layers formed on n-type $6 \mathrm{H}-\mathrm{SiC}(0001)$ and additional graphene layers were formed on them by the MBE. Details of the graphene formation and the determination of its layer number are described elsewhere [10]. Prior to the growth, the substrate was annealed at $550^{\circ} \mathrm{C}$ for one hour under a vacuum of less than $10^{-8}$ Torr. For the growth, the substrate was first heated to around $620^{\circ} \mathrm{C}$ and the $\mathrm{W}$ filament temperature was set at $2000^{\circ} \mathrm{C}$. Then, the supply of ethanol gas and the growth were started. During the growth, the pressure was $4 \times 10^{-5}$ Torr. The total growth time was six hours. The growth system is also equipped 
with a monochromatized $\mathrm{Al} \mathrm{K} \alpha$ source $(1486.6 \mathrm{eV})$ and a photoelectron analyzer. Using x-ray photoelectron spectroscopy (XPS) system, we performed in situ measurements. After the MBE growth, the number of graphene layers, estimated by the photoelectron attenuation of $\mathrm{x}$ ray photoelectron spectroscopy (XPS), increased by about four. Further details of the graphene growth are described in $[8]$.

After the growth, the substrate was removed from the growth system and about $100 \mathrm{~nm}$ of amorphous carbon thin film was deposited on the sample to capture TEM images. X-ray reflectivity was measured on this sample using the hard x-ray $(12 \mathrm{keV})$ at the beamline of BL46XU of SPring-8 and the CTR scattering spectrum was obtained. During the reflectivity measurements, the sample was covered with a transparent dome made of kapton, which was filled with atmospheric He gas. Even in this situation, the exposed graphene layer was easily destroyed, perhaps due to the plasma of residual gas, such as oxygen and nitrogen, created by the intense x-ray. Thus, the amorphous carbon was needed to measure the reflectivity and served as a protective covering to prevent the degradation of graphene layers.

\section{RESULTS AND DISCUSSION}

\section{A. Macroscopic thickness of graphene before MBE growth}

The initial graphene thickness was three to four layers, [8] which was determined by low-energy electron microscope (LEEM) observation [10]. This thickness was determined for the microscopic specific area of the substrate center and appropriate value for discussing the structure and thickness observed by TEM. However, for the x-ray reflection measurement, the size of its observed area was several millimeters in the longitudinal direction of the $\mathrm{x}$ ray beam and far larger than the LEEM observation area. Hence, the thickness of the graphene layer before the MBE growth was estimated by XPS, a macroscopic method, using $\mathrm{C}$ 1s core-level photoelectron spectra.

As a reference, graphene formed by the thermal decomposition of $\mathrm{SiC}$ at substrate temperature of $1820^{\circ} \mathrm{C}$ and Ar pressure of 90 Torr was used. Atomic force microscopy (AFM) topographic (height) and phase images are shown
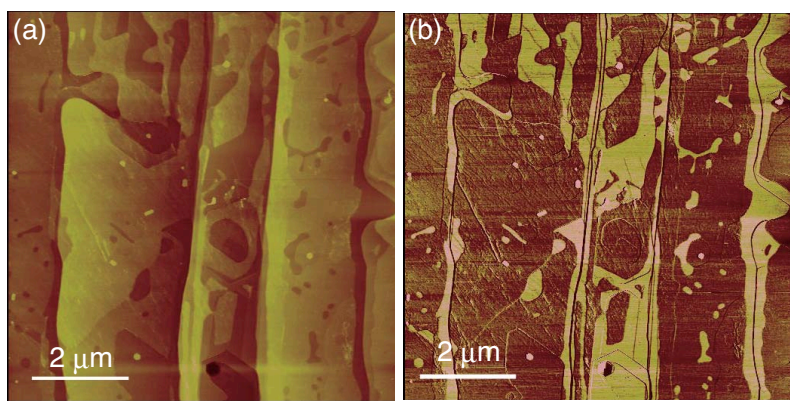

FIG. 1: AFM (a) Topographic (height) and (b) phase images of the reference sample, which was grown by thermal decomposition of $\mathrm{SiC}$ with an infrared heating system.

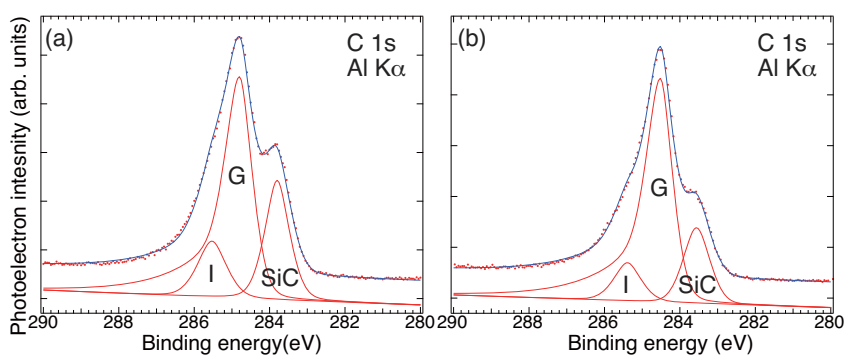

FIG. 2: C 1s core-level photoelectron spectra of (a) the reference sample and (b) the sample used in this study before the MBE growth. Dots are experimental data and fitting results are indicated by lines. The components corresponding, respectively, to bulk $\mathrm{SiC}$, graphite/graphene, and the interface (buffer) layers $(6 \sqrt{3} \times 6 \sqrt{3})$ are denoted as $\mathrm{SiC}, \mathrm{G}$, and I.

in Fig. 1. This graphene was formed by using an infrared heating system, which can uniformly heat a whole substrate whose size is $1 \mathrm{~cm}$ square. In the phase images, areas with two or more layers of graphene appear brighter than areas with monolayer graphene [11]. In the phase image, the brighter area occupies $25.2 \%$ of the whole image. From this value, we estimated the graphene thickness of this sample to be 1.25 monolayers (MLs), assuming brighter area indicates two MLs. The $\mathrm{C}$ 1s core-level photoelectron spectrum of this reference sample is shown in Fig. 2(a). Applying a curve-fit procedure shows that the $\mathrm{C}$ 1s spectra have three components [12] located at binding energies of 283.8, 284.8, and $285.5 \mathrm{eV}$. These components corresponding respectively to bulk $\mathrm{SiC}$, graphite/graphene, and the interface (buffer) layers $(6 \sqrt{3} \times 6 \sqrt{3})$ are denoted as $\mathrm{SiC}, \mathrm{G}$, and I in Fig. 2(a).

Figure 2(b) shows the $\mathrm{C}$ 1s spectrum of the sample used in this study before the MBE growth. The three components obtained by the fitting are shown below the spectrum. Here, when the photoelectron intensities of graphene component $I_{\mathrm{G}}$ and $\mathrm{SiC}$ substrate component $I_{\mathrm{SiC}}$ are used, the thickness of graphene $d$ is calculated by the following equations:

$$
\begin{gathered}
d=\left(\lambda_{1} \sin \theta\right) \ln (A X+1) \\
X=\frac{I_{\mathrm{G}}}{I_{\mathrm{SiC}}}
\end{gathered}
$$

where $\lambda_{1}, \theta$, and $A$ represent the photoelectron mean free path (MFP) of graphene, the takeoff angle of photoelectrons, $25^{\circ}$ in this experiment, and a constant introduced using densities of carbon atoms, the MFP of $\mathrm{C}$ 1s photoelectrons, and the yield of $\mathrm{C}$ 1s photoelectrons for graphene and $\mathrm{SiC}$ substrate. Because the graphene thickness of the reference sample is known to be the value mentioned above, when we use the intensities of the two components in Fig. 2(a) and assume $\lambda_{1} 21.3 \AA[13], A$ can be calculated. Then, using this value of $A$, we can calculate the thickness of the graphene of the sample used in this study before the MBE growth, and the value is 1.79 MLs when the intensities of the two components in Fig. 2(b) are used. This indicates that the average graphene thickness is about two MLs for the observation 
area of the x-ray reflection measurement on the sample before the MBE growth.

\section{B. X-ray reflectivity measurement after $\mathrm{MBE}$ growth}

To obtain detailed information about the graphene layers, we measured $\mathrm{x}$-ray reflectivity for the sample grown by MBE. The data were collected by integrating rocking curves around $q_{\|}=0$ for different perpendicular momentum-transfer vectors, $q_{\perp}=2 \pi \ell / c_{S i C}$, where $\mathbf{q}=$ $\mathbf{k}_{f}-\mathbf{k}_{i}$. This measurement geometry is shown in Fig. 3 . To analyze the data, we used a calculation model similar to the one demonstrated by Hass et al. [14, 15]. The structural model we used is shown in Fig. 4. Hass et al. assumed graphene layers are uniform and their spacing is equal. Therefore, they set average values for graphene layers. We extended their model to fit our situation by setting the parameters for each graphene layer instead.

Using the structural model, the scattered x-ray intensity $I(\Theta, \ell)$ can be calculated by the sum of three scattered amplitudes: the bulk $F_{\text {bulk }}$, the interface region $F_{\mathrm{I}}$, and the graphene $F_{\mathrm{G}}$.

$$
I(\Theta, \ell)=A(\Theta, \ell)\left|\frac{F_{\text {bulk }}(\ell)}{1-e^{-2 \pi i \ell}}+F_{\mathrm{I}}(\ell)+\frac{\rho_{\mathrm{G}}}{\rho_{\mathrm{SiC}}} F_{\mathrm{G}}(\ell)\right|^{2} .
$$

$A(\Theta, \ell)$ is as term that contains all corrections due to the experimental geometry. The ratio of the areal densities of the $\mathrm{SiC}(0001)$ and a graphene (0001) plane, $\rho_{\mathrm{G}} / \rho_{\mathrm{SiC}}=3.132$, is used to properly normalize the scattered amplitude from the graphene layer per $\mathrm{SiC}(0001)$ unit cell.

The structure factor of the interface region between bulk and the graphene film corresponding to $F_{\mathrm{I}}(\ell)$ in Eq. (3) can be written as

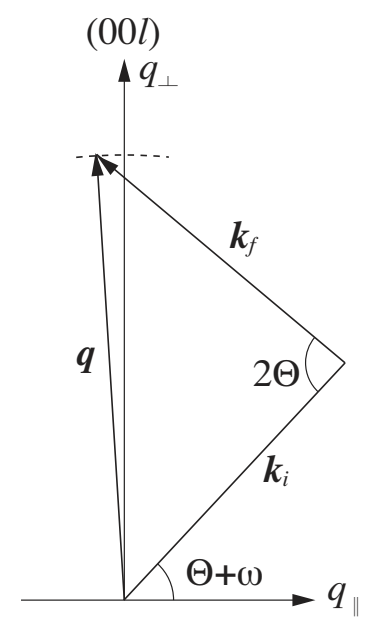

FIG. 3: Schematic drawing of the reflectivity geometry. Incident wave $\mathbf{k}_{i}$ strikes the sample surface at an angle $\Theta+\omega$. The diffracted wave $\mathbf{k}_{f}$ is kept fixed at $2 \Theta$ from $\mathbf{k}_{i}$. q is "rocked" through the $(00 \ell)$ rod by rotating the sample through an angle $\pm \omega$.

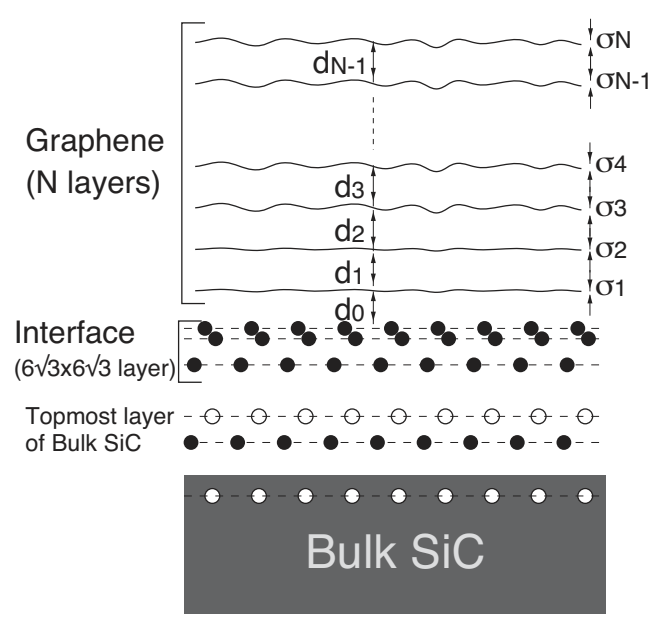

FIG. 4: Schematic model of $\mathrm{N}$ layers of graphene grown on $\mathrm{SiC}$ substrate. Filled and open circles respectively indicate carbon and $\mathrm{Si}$ atoms.

$$
F_{\mathrm{I}}(\ell)=\sum_{j=1}^{5} f_{j}(\ell) \rho_{j} e^{i 2 \pi \ell z_{j} / c_{\mathrm{SiC}}},
$$

where $\rho_{j}$ is relative atom density for the $j$ th interface layer at a vertical position $z_{j}$ and $f_{j}(\ell)$ is the atomic form factor of $\mathrm{C}$ or $\mathrm{Si}$. This interface structure between $\mathrm{SiC}$ and the graphene is assumed to consist of five layers. In this study, we examined the fitting only for the carbon atom model (one Si layer and four C layers) because the best reflectivity fit to the data is nearly identical for the other models including additional Si layer described in the reference [14] and because we believe that this model is the most probable considering the growth model of graphene from $\mathrm{SiC}$. [16]

Since the lateral distribution of graphene layers varies, we defined occupancy parameter $p_{n}$ as the fractional surface area for each graphene layer. Then, the multilayer graphene structure factor can be written in the general form

$$
\begin{gathered}
F_{\mathrm{G}}(\ell)=f_{C}(\ell) \sum_{n=1}^{N} p_{n} F_{n}(\ell)\left\{\sum_{m=1}^{n} e^{2 \pi i \ell z_{m} / c_{\mathrm{G}}}\right\}, \\
z_{m}=\sum_{n=0}^{m-1} d_{n}, \\
F_{n}(\ell)=e^{-\left(2 \pi \ell / c_{\mathrm{SiC}}\right)^{2} \sigma_{n}^{2} / 2},
\end{gathered}
$$

where $f_{c}$ is the atomic form factor for carbon and $N$ is the number of layers of the graphene. The $d_{n}$ is the spacing between the graphene layer and the underlying atomic layer as shown in Fig. 4. $F_{n}(\ell)$ is a structural factor for a small vertical height distribution in each graphene layer and $\sigma_{n}$ is assumed for a random vertical disorder of each layer. We did not use the average value; instead, we independently set $d_{n}$ for each graphene layer. This is our 


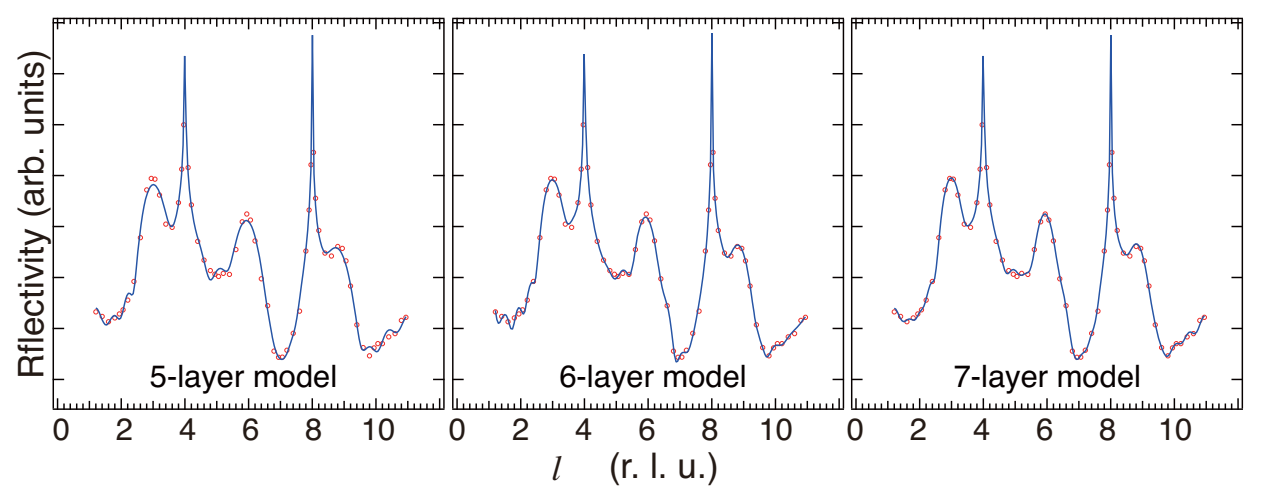

FIG. 5: Specular reflectivity versus $q_{\perp}$ (in r.l.u.) for the MBE-grown graphene sample. Open circles are the experimental data. Solid lines are the best-fit results when five (left), six (center), and seven (right) of graphene layers were assumed.

Hass-model [14, 15] modification for obtaining the information about graphene layer grown by MBE.

Reflectivity data for the MBE-grown graphene sample are shown in Fig. 5. Open circles are the data calculated by integrating rocking curves. The three panels in Fig. 5 contain the same experimental data. For each panel, the best-fit result is also indicated by a line when five, six, and seven layers of graphene are assumed. In addition to sharp prominent peaks from the $\mathrm{SiC}$ bulk, broad peaks were observed at $\ell$ of 3,6 and 9 . These peaks, which could not be detected using a laboratory x-ray source, are derived from graphene.

By comparing the three fitting result, especially looking at the intensities of the broad peaks derived from graphene, we found that seven layers of graphene best reproduce the x-ray reflectivity data (right panel in Fig. 5). Table I lists the fitting parameters for the seven-layer model. Because the initial graphene layer formed by thermal decomposition of $\mathrm{SiC}$ in LEEM apparatus is about two MLs, parameters for the first and second layer in Table I correspond to those for the initial graphene layer. Consequently, the parameters of the third to seventh graphene layers correspond to those of the MBE grown graphene layer.

\section{Discussion of MBE-grown graphene}

From Table I, the average of the spacing of the MBEgrown graphene is $3.39 \AA$, which is the average of inter-

TABLE I: Best-fit structural parameters for the MBE-grown sample.

\begin{tabular}{cccc}
\hline \hline $\begin{array}{c}N \text { th } \\
\text { graphene layer }\end{array}$ & $\begin{array}{c}d_{n-1}: \\
\text { spacing }(\AA)\end{array}$ & $\begin{array}{c}p_{n}: \\
\text { occupancy }\end{array}$ & $\begin{array}{c}\sigma_{n}: \\
\text { vertical disorder }\end{array}$ \\
\hline 1 & 1.89 & 1.019 & 0.101 \\
2 & 3.35 & 0.932 & 0.130 \\
3 & 3.38 & 0.652 & 0.152 \\
4 & 3.38 & 0.670 & 0.194 \\
5 & 3.42 & 0.365 & 0.197 \\
6 & 3.37 & 0.237 & 0.211 \\
7 & 3.42 & 0.093 & 0.145 \\
\hline \hline
\end{tabular}

layer spacing values for bulk crystalline graphite $(3.354 \AA)$ and turbostratic graphite $(3.440 \AA)$ [15]. The spacing of the initial graphene layer is $3.35 \AA$, which is nearly identical to that of bulk crystalline graphite. These results indicates that the atomic arrangement of the MBEgrown graphene layer is not well aligned to the underlying graphene layer due to weak interlayer interaction, suggesting that its atomic arrangement rotates in plane, although the atomic arrangement of graphene remains. This rotation of atomic arrangement is consistent with a reflection high-energy electron diffraction (RHEED) observation of another sample on which about one ML of graphene had been grown at the same substrate temperature [9]. In addition, the height distributions of the MBE-grown graphene are larger than that of the initial graphene, indicating that the graphene sheet of the initial graphene is flatter and its quality is better than the MBE-grown graphene.

The occupancies of the MBE-grown graphene are smaller than unity. This is in contrast to the occupancies of the initial graphene, which are nearly unity. Additionally, they are smaller than that of their underlying graphene. This trend indicates that, although each layer grew laterally, the graphene grew in a three-dimensional (3D) manner by the formation of multilayer islands, which were formed by the nucleation of the second layer on top of monolayer islands before coalescence [17]. Moreover, the sum of the occupancies of the MBE-grown graphene is about two MLs. This is half of the layer number estimated by XPS. This discrepancy implies that a turbostratic phase appears on graphene of such thickness, because the CTR scattering results reflect only layered structures. This result is consistent with a RHEED observation, in which a ring pattern image was observed after the MBE growth.

The results indicate that the initial several layers of graphene form a layered structure, which suggests our growth method is feasible for FLG formation. However, the graphene grew in a 3D mode, indicates that the growth temperature is not high enough for layer-bylayer growth because the short diffusion length of growth species at low temperature induces the multilayer growth mode [17]. Therefore, a higher substrate temperature than that of present study, $620^{\circ} \mathrm{C}$, is needed for single layer graphene or FLG to completely covered the sub- 
strate by layer-by-layer growth. This high-temperature growth would also improve the quality of the graphene grown by MBE, which could decrease the misalignment of the atomic arrangement between graphene sheets and improve their flatness.

\section{SUMMARY}

For graphene formation, we proposed in a previous study a new method based on gas-source MBE, in which a cracked-ethanol source is employed, and showed the feasibility of this growth method. To evaluate the graphene grown by our new method, from the macroscopic viewpoint, we investigated CTR scattering by x-ray reflectivity measurement in the present study. From the analysis of $\mathrm{x}$-ray reflectivity data, we found that a seven-layer structural model reproduces the experimental data, indicating that the topmost five layers form a layered structure from the macroscopic view, also. The average of the spacing of the MBE-grown graphene was $3.39 \AA$, which is the average of interlayer spacing values for bulk crystalline graphite and turbostratic graphite. The height distributions of the MBE-grown graphene were relatively large, indicating that undulation of the graphene sheet was rel- atively large. The occupancies of the graphene suggest that graphene did not grow in a layer-by-layer manner but instead grew in a 3D manner, while each layer grew laterally. Moreover, the sum of the occupancies was about two MLs, which is half of the layer number estimated by XPS. This discrepancy implies that a turbostratic phase appears on the graphene. These results indicate that our new approach is feasible for the formation of wafer-scale graphene, although the quality of the present MBE-grown graphene is not high. The $3 \mathrm{D}$ growth mode and the in plane rotation of the atomic arrangement of the graphene sheet are issues to be resolved. We believe that optimization of growth condition, especially a high growth temperature, will improve the quality of the graphene.

\section{Acknowledgments}

The synchrotron radiation experiments were performed at the BL46XU of SPring-8 with the approval of the Japan Synchrotron Radiation Research Institute (JASRI) (Proposal No. 2008A1822 and 2008B1949). This work was partially supported by the Japan Society for the Promotion of Science (JSPS) KAKENHI (21246006, 22310077).
[1] K. S. Novoselov, A. K. Geim, S. V. Morozov, D. Jiang, Y. Zhang, S. V. Dubonos, I. V. Grigorieva, and A. A. Firsov, Science 306, 666 (2004).

[2] A. J. van Bommel, J. E. Crombeen, and A. van Tooren, Surf. Sci. 48, 463 (1975).

[3] C. Oshima and A. Nagashima, J. Phys.: Condens. Matter 9, 1 (1997).

[4] L. C. Isett and J. M. Blakely, Surf. Sci. 58, 397 (1976).

[5] C. Riedl, C. Coletti, T. Iwasaki, A. A. Zakharov, and U. Starke, Phys. Rev. Lett. 103, 246804 (2009).

[6] B. Premlal, M. Cranney, F. Vonau, D. Aubel, D. Casterman, M. M. De Souza, and L. Simon, Appl. Phys. Lett. 94, 263115 (2009).

[7] K. S. Kim, Y. Zhao, H. Jang, S. Y. Lee, J. M. Kim, K. S. Kim, J.-H. Ahn, P. Kim, J. Y. Choi and B. H. Hong, Nature 457, 706 (2009).

[8] F. Maeda and H. Hibino, Phys. Status Solidi B 247, 916 (2010).

[9] F. Maeda and H. Hibino, Jpn. J. Appl. Phys. 49, 04DH13 (2010).
[10] H. Hibino, H. Kageshima, F. Maeda, M. Nagase, Y. Kobayashi, and H. Yamaguchi, Phys. Rev. B 77, 075413 (2008).

[11] S. Tanabe, Y. Sekine, H. Kageshima, M. Nagase, and H. Hibino, Appl. Phys. Express 3, 075102 (2010).

[12] C. Virojanadara, M. Syvajarvi, R. Yakimova, and L. I. Johansson, A. A. Zakharov and T. Balasubramanian, Phys. Rev. B 78, 245403 (2008).

[13] J. Zemek, J. Potmesil, M. Vanecekm B. Lesiak, and A. Jablonski, Appl. Phys. Lett. 87, 262114 (2005).

[14] J. Hass, J. E. Millan-Otoya, P. N. First, and E. H. Conrad, Phys. Rev. B 78, 205424 (2008).

[15] J. Hass, R. Feng, J. E. Millan-Otoya, X. Li, M. Sprinkle, P. N. First, W. A. de Heer, E. H. Conrad, and C. Berger, Phys. Rev. B 75, 214109 (2007).

[16] H. Kageshima, H. Hibino, M. Nagase, and H. Yamaguchi, Appl. Phys. Express 2, 065502 (2009).

[17] J. Tersoff, A. W. Denier van der Gon, and R. M. Tromp, Phys. Rev. Lett. 72, 266 (1994). 